\title{
CES
}

COOPERATIVISMO E ECONOMÍA SOCIAL

Núm. 43 (2020-2021), páxs. 315-322

ISSN: 2660-6348

\section{NUEVOS MECANISMOS PARA FACILITAR EL EJERCICIO DE LOS DERECHOS DE PARTICIPACIÓN EN LA TOMA DE DECISIONES EN LAS COOPERATIVAS GALLEGAS}

\section{NEW MECHANISMS TO FACILITATE THE EXERCISE OF PARTICIPATION RIGHTS IN DECISION- MAKING IN GALICIAN COOPERATIVES"}

Manuel José VÁzQuez PenA*

Profesor Titular de Derecho Mercantil de la Universidad de A Coruña. Dirección de correo electrónico: manuel.jose.vazquez.pena@udc.es 


\title{
RESUMO
}

El Legislador gallego, a través de la Ley 4/2021, de 28 de enero, "de medidas fiscales y administrativas", introduce distintas modificaciones en la Ley 5/1998, de 18 de diciembre, de "Cooperativas de Galicia", con la intención de habilitar mecanismos que faciliten el ejercicio de los derechos de participación en la toma de decisiones societarias por parte de las personas socias, más allá de la mera consolidación de medidas tendentes a la flexibilización del desarrollo de las reuniones por medios telemáticos.

Palavras Clave: Sociedades Cooperativas, Asamblea general, reuniones por medios telemáticos, Asamblea general de delegados, pandemia, medidas por las restricciones impuestas en materia de circulación y de reunión.

\begin{abstract}
The Galician Legislator, through Law 4/2021, of January 28, "on fiscal and administrative measures", introduces different modifications in Law 5/1998, of December 18, on "Cooperatives of Galicia", with the intention to enable mechanisms that facilitate the exercise of participation rights in corporate decision-making by members, beyond the simple consolidation of measures aimed at making the development of meetings more flexible by telematic means.

Keyworlds: Cooperative societies, General Assembly, meetings by telematic means, General Assembly of delegates, pandemic, measures due to restrictions imposed on movement and assembly.
\end{abstract}


SUMARIO: I. NOTA INTRODUCTORIA. II. LAS MODIFICACIONES DE LOS ARTÍCULOS 35, 36 Y 39 DE LA LEY DE COOPERATIVAS DE GALICIA. III. LAS NUEVAS DISPOSICIONES ADICIONALES DÉCIMA Y DÉCIMOPRIMERA DE LA LEY DE COOPERATIVAS DE GALICIA.

SUMMARY: I. INTRODUCTORY NOTE. II. THE AMENDMENTS TO ARTICLES 35, 36 AND 39 OF THE LAW OF COOPERATIVES OF GALICIA. III. THE NEW ADDITIONAL TENTH AND ELEVENTH PROVISIONS OF THE LAW OF COOPERATIVES OF GALICIA.

\section{NOTA INTRODUCTORIA}

$\mathrm{E}$ Legislador gallego, a través de la Ley 4/2021, de 28 de enero, "de me-

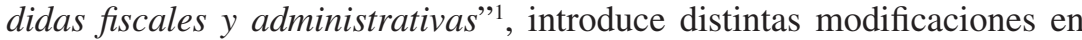
la Ley 5/1998, de 18 de diciembre, de "Cooperativas de Galicia". En un contexto de pandemia que no permite aún vislumbrar una total vuelta a la normalidad, y probablemente en previsión de la existencia de acontecimientos futuros que aconsejen actuar de la misma o similar manera, el Legislador gallego trata con estas modificaciones de habilitar mecanismos que faciliten el ejercicio de los derechos de participación en la toma de decisiones societarias por parte de las personas socias, más allá de la mera consolidación de medidas tendentes a la flexibilización del desarrollo de las reuniones por medios telemáticos ${ }^{2}$.

Este es el planteamiento de partida de las modificaciones introducidas en la Legislación cooperativa gallega, siguiendo la tendencia que, en mayor o menor medida, se ha plasmado en otras Legislaciones del Estado español ${ }^{3}$, o incluso en

\footnotetext{
BOE del 25 de marzo de 2021.

2 Medidas tendentes a la flexibilización del desarrollo de las reuniones por medios telemáticos implantadas con carácter temporal en el varias veces modificado Real Decreto-ley 8/2020, de 17 de marzo, "de medidas urgentes extraordinarias para hacer frente al impacto económico y social del COVID-19" (BOE del 18 de marzo).
}

3 Pueden consultarse, por ejemplo, la Orden de 5 de marzo 2021, de la Consejería de Economía, Empleo y Competitividad, "por la que se habilita la legalización telemática de los libros obligatorios de las cooperativas en el Registro de Cooperativas de la Comunidad de Madrid" (BO. Comunidad de Madrid de 18 de marzo de 2021), en la Comunidad de Madrid; el Decreto-ley 8/2020, de 26 de junio, "de medidas excepcionales para facilitar la convocatoria y reunión de los órganos sociales y la eficacia de los acuerdos de las entidades cooperativas valencianas" (BOE del 13 de agosto de 2020), en la Comunidad Valenciana; el Decreto-ley 7/2021, de 9 de febrero, de "medidas extraordinarias en materia de cooperativas, empresas y entidades de la economía social como consecuencia de la crisis derivada de la COVID-19 y de modificación del Decreto ley 16/2020, de 5 de mayo de 2020, de medidas urgentes complementarias en materia de transparencia, ayudas de carácter social, contratación y movilidad para hacer frente a la COVID-19" (BOE del 19 de marzo de 2021), o el 
el ámbito de la Unión Europea ${ }^{4}$, como consecuencia de las especiales circunstancias que han marcado el día a día en los últimos meses y que, de momento, siguen definiendo el contexto actual.

Así las cosas, el artículo 26, uno de los que integran el Capítulo VII ("Empleo e igualdad") de la Ley gallega 4/2021, modifica a lo largo de sus cinco apartados los artículos 35, 36 y 39 de la Ley de Cooperativas de Galicia, añadiendo también a la misma dos disposiciones adicionales, la décima y la decimoprimera.

\section{LAS MODIFICACIONES DE LOS ARTículos 35, 36 Y 39 DE LA LEY de Cooperativas de Galicia}

El artículo 35 ("Constitución y funcionamiento de la asamblea") es modificado en sus números 1, 2 y 3 . En el primero, sin romper la tradicional previsión de que "la asamblea general tendrá lugar en la localidad donde radique el domicilio social de la cooperativa", se establecen dos excepciones más a las dos hasta este momento existentes, y que se mantienen en similares términos: a) Aquel supuesto en el que la asamblea general tenga el carácter de universal; y b) aquel otro en el que los estatutos, en los casos en que exista causa que lo justifique, fijen, con carácter general, otros lugares de reunión o los criterios que seguirá el órgano de administración para la determinación del lugar de celebración de la asamblea. Con la Ley 4/2021 se añaden dos nuevas excepciones:

a) La posibilidad de que la asamblea general "se realice de forma telemática"; y

b) aquellos casos en los que, "por circunstancias sobrevenidas, en la localidad donde radique el domicilio social de la cooperativa no exista un local que permita el normal desarrollo de la asamblea en condiciones de seguridad'. El órgano de administración, cuando sea aconsejable esta posibilidad, "tendrá que motivar suficientemente las circunstancias que aconsejen esta medida, "así como la idoneidad de la localidad escogida en términos de facilidad de comunicación respecto de la localidad donde la cooperativa tiene el domicilio social o donde reside la mayoría de las personas socias".

Se trata, en definitiva, con esta nueva redacción, con esta nueva excepción a la regla general, de facilitar la realización de reuniones presenciales en condiciones

Decreto-ley 47/2020, de 24 de noviembre, de "medidas extraordinarias de carácter económico en el sector de las instalaciones juveniles, de medidas en el sector de las cooperativas y de modificación del Decreto ley 39/2020, de 3 de noviembre de 2020, de medidas extraordinarias de carácter social para hacer frente a las consecuencias de la COVID-19, y del Decreto ley 42/2020, de 10 de noviembre de 2020, de medidas urgentes de apoyo a entidades del tercer sector social" (BOE del 19 de enero de 2021), ambos en la Comunidad de Cataluña.

4 Reglamento (UE) 2020/699, de 25 de mayo, de "medidas temporales relativas a la junta general de las sociedades europeas (SE) y la asamblea general de las sociedades cooperativas europeas (SCE)" (DOL del 27 de mayo de 2020). 
de seguridad, habilitando la opción de utilizar instalaciones disponibles en localidades próximas, cuando no existan locales adecuados en la localidad en que la cooperativa tenga su domicilio social.

El nuevo número 2 del artículo 35, en realidad, no supone ninguna novedad por cuanto aúna en un único precepto lo que decía el anterior número 2 más el primer párrafo del antiguo número 3 , eso sí, con alguna pequeña modificación en la redacción. Es probable que esta nueva "reorganización de contenidos" en este apartado del precepto responda simplemente a una mejor exposición global, en la que se implemente con más coherencia las nuevas redacciones de los números 1 y 3 del artículo 35.

Mayor novedad aporta la actual redacción del número 3 del artículo 35 de la Ley de Cooperativas de Galicia, consagrando, sin necesidad de previsión estatutaria, el desarrollo de las reuniones de la asamblea general a través de videoconferencia u otros medios telemáticos que permitan la participación a distancia, bien de manera íntegra bien de manera mixta, es decir, presencial y por medios telemáticos. En cualquier caso, añade el precepto que ha de garantizarse que "todas las personas que deban ser convocadas tengan posibilidad de acceso". Será en la convocatoria donde se determinen "los plazos, las formas y los modos de ejercicio de los derechos por parte de las personas que asistan telemáticamente a la asamblea".

Sea como fuere, cuando se convoquen reuniones de la asamblea general para su realización por medios telemáticos, el órgano de administración deberá adoptar las medidas necesarias para garantizar: a) La verificación de la identidad de las personas asistentes; b) la acreditación de la representación con que actúan, en su caso; c) el ejercicio del derecho de participación con voz y voto, según proceda; d) el sentido de los votos emitidos y su cómputo, teniendo en cuenta el voto plural cuando proceda; y e) el secreto de los votos, cuando deban tener tal carácter.

Aunque es indudable que estas medidas, establecidas en el nuevo artículo 35, mejoran notablemente las posibilidades para la celebración de reuniones en condiciones de seguridad, lo cierto es que no bastan para garantizar en todos los casos la efectiva participación de las personas socias, habida cuenta de la brecha digital que subsiste entre la población más joven y urbana, de una parte, frente a la población de edad más avanzada y del ámbito rural, de otra.

Obviamente, sin perjuicio de que con esta norma se consoliden estas medidas en el ámbito cooperativo gallego, una solución válida y factible para muchas cooperativas, especialmente las de menor tamaño, la realidad demuestra la necesidad de establecer otros mecanismos para aquellas otras cooperativas en las que el elevado número de personas socias, la avanzada edad de estas y el hecho de que residan en entornos rurales, pueden dificultar el acceso a la tecnología. 
Por ello, además de la posibilidad apuntada, esto es, de la flexibilización del desarrollo de las reuniones por medios telemáticos, en la Ley 4/2021 se recogen otras medidas que facilitan la participación en la toma de decisiones societarias por medio de terceras personas, como son la designación de representantes y las asambleas de delegados, soluciones que permiten reducir de forma muy significativa el número de personas asistentes presencialmente en las reuniones o que deban tener acceso a los medios tecnológicos para participar en las mismas.

El nuevo número 8 del artículo 36 ("Derecho de voto") se refiere a la primera posibilidad, a la posibilidad de que las personas socias puedan hacerse representar por otras personas socias, aunque $-y$ he aquí la novedad- los estatutos no lo prevean. A diferencia de lo que ocurría antes de la modificación, estableciéndose el límite en dos representaciones, en la actualidad una misma persona podrá actuar en representación de hasta diez personas socias.

En caso de que la persona representante asista a la reunión de forma presencial, la representación se acreditará mediante autorización por escrito autógrafo o poder especial suscrito por el representado o representada. En congruencia con el nuevo artículo 35, en caso de que participe por medios telemáticos, la convocatoria deberá establecer la forma en que se debe acreditar la representación.

Sea como fuere, tal como se contemplaba también en la anterior redacción del precepto, "la representación debe otorgarse para cada asamblea, y debe ser verificada por el órgano de intervención de la cooperativa o, en su defecto, por la presidencia y la secretaría".

Por su parte, el nuevo número 1 del artículo 39 ("Asamblea general de delegados") alude a la segunda posibilidad, admitiendo, como en la anterior redacción, que "los estatutos podrán prever que las competencias de la asamblea general se ejerzan mediante una asamblea de segundo grado, integrada por los delegados designados en juntas preparatorias".

Ha de destacarse, no obstante, y he aquí el cambio legislativo, que, aunque no lo prevean los estatutos (y antes era absolutamente necesario), "también se podrá aplicar esta modalidad cuando en una cooperativa concurran circunstancias que dificulten la presencia simultánea de todas las personas socias en la asamblea general". No existiendo previsión estatutaria al respecto, será el órgano de administración el encargado de justificar "de forma suficiente esta circunstancia", así como de establecer "los criterios de adscripción de las personas socias a las juntas preparatorias, que se comunicarán junto con la convocatoria". 


\section{LAS NUEVAS DISPOSICIONES ADICIONALES DÉCIMA Y DÉCIMOPRIMERA DE LA LEY DE COOPERATIVAS DE GALICIA}

Con independencia de que el antes citado Real Decreto-ley 8/2020, de 17 de marzo, estableciese disposiciones específicas en cuanto al plazo para la adopción de acuerdos relativos a las cuentas anuales, la realidad de los últimos meses, particularmente durante la vigencia del estado de alarma ${ }^{5}$, demostró que existían numerosas decisiones que debían tomarse en un plazo determinado y cuya adopción se vio sin duda condicionada por las restricciones impuestas en materia de circulación y de reunión. Así las cosas, los hechos hicieron patente la necesidad de establecer un plazo para que estas decisiones pudiesen llevarse a cabo en aplicación de las nuevas medidas aprobadas por la ahora comentada la Ley 4/2021, de 28 de enero.

En este entendimiento, tiene sentido la redacción de una nueva disposición adicional, como es el caso de la décima ("No existencia de quorum en segunda convocatoria"), al disponer ésta que "excepcionalmente, hasta el 31 de diciembre de 2021, y con independencia de la modalidad de realización de la reunión, las asambleas generales de las cooperativas a las que le sea de aplicación la Ley 5/1998, de 18 de diciembre, de cooperativas de Galicia, quedarán válidamente constituidas en segunda convocatoria cualquiera que sea el número de cooperativistas presentes o representados, aunque los estatutos no lo hayan establecido".

Con similar razonamiento y prácticamente idénticos motivos, la imposibilidad de realización de asambleas durante el estado de alarma y los distintos estadios de la nueva normalidad hace necesario, en beneficio de la seguridad jurídica y para no comprometer la capacidad de obrar de la sociedad cooperativa, prolongar la vigencia por el tiempo mínimo imprescindible de aquellos cargos cuyo nombramiento haya caducado durante este período de tiempo. En este sentido, la nueva disposición adicional decimoprimera ("Plazo para la realización de asambleas generales y prórroga de los nombramientos") contiene tres previsiones:

a) En primer lugar, se dispone que las asambleas generales de las cooperativas a las que sea de aplicación la Ley 5/1998, de Cooperativas de Galicia, que debieran tener lugar en una fecha posterior al 14 de marzo de 2020 y que no se pudieron realizar en cumplimiento de las medidas dictadas para hacer frente a la pandemia de la COVID-19, podrán celebrarse con fecha límite del 31 de diciembre de 2021.

\footnotetext{
5 Real Decreto 926/2020 de 25 de octubre, del Ministerio de la Presidencia, Relaciones con las Cortes y Memoria Democrática, "por el que se declara el estado de alarma para contener la propagación de infecciones causadas por el SARS-COV-2" (BOE del 25 de octubre de 2020); prorrogado mediante el Real Decreto 956/2020, de 3 de noviembre (BOE del 4 de noviembre de 2020).
} 
b) En segundo lugar, prevé la disposición adicional que aquellos nombramientos para cargos de los órganos sociales de las cooperativas a las que sea de aplicación la citada Ley 5/1998, cuya vigencia haya finalizado después del 14 de marzo de 2020, se entenderán prorrogados hasta el 31 de diciembre de 2021, sin perjuicio de que puedan ser renovados o separados en cualquier momento anterior por el órgano competente.

c) Finalmente, en tercer lugar, se señala que, con independencia de lo que dispongan sus estatutos, las modificaciones apuntadas en el punto anterior serán aplicables directamente a las cooperativas gallegas. No obstante, cuando se pretenda modificar los estatutos vigentes, continúa la disposición, estos deberán adaptarse íntegramente a lo dispuesto en la Ley de Cooperativas de Galicia. 